\title{
Pre-emptive CMV therapy versus universal CMV prophylaxis in renal transplant recipients
}

A new study reports that of the two main strategies for the prevention of cytomegalovirus (CMV) in renal transplant recipients, pre-emptive treatment of CMV may be associated with better outcomes than universal CMV antiviral prophylaxis.

Tomas Reischig and co-workers used a randomized, open-label trial to

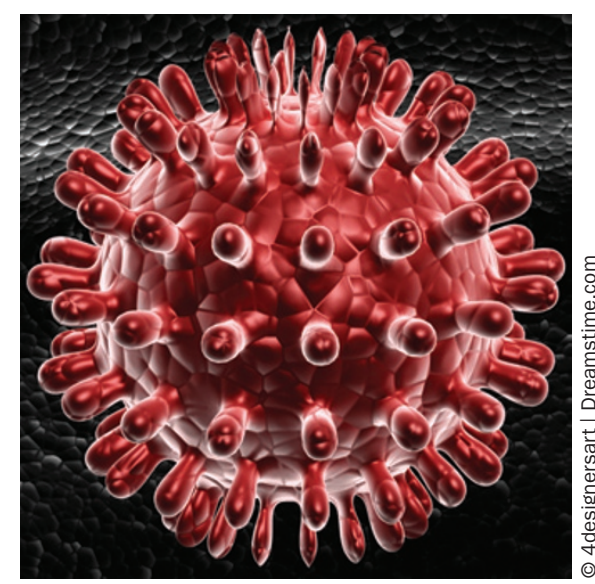

compare these two strategies in 70 renal transplant recipients among whom either the recipient or the donor was CMVseropositive. Patients were randomly assigned to either universal prophylaxis with valacyclovir for 3 months $(n=34)$ or to pre-emptive therapy with valganciclovir when CMV viraemia of $\geq 2,000$ copies per $\mathrm{ml}$ was detected $(n=36)$.

Protocol biopsies were performed in 55 patients at 3 years after transplantation. At this point, interstitial fibrosis and tubular atrophy was more common in biopsy samples from the prophylaxis group than in samples from the preemptive therapy group (38\% versus $19 \%$, respectively).

Universal prophylaxis was associated with worse 4-year allograft survival than was pre-emptive therapy ( $74 \%$ versus $92 \%)$, despite an increased incidence of acute cellular rejection episodes in the preemptive therapy group at 1 year. The worse 4-year survival in the prophylaxis group was mostly a result of patients experiencing late-onset asymptomatic CMV viraemia after prophylaxis completion. The researchers also found increased intrarenal mRNA expression of genes involved in fibrogenesis in the prophylaxis group.

"Our study suggests that it is not possible to limit the control of CMV to the shortterm post-transplantation period and that a regimen that improves recovery of the CMV-specific immune response-which can control CMV replication in the long term-is needed," states Reischig. "It is presumed that early low-grade episodes of CMV viraemia controlled by preemptive valganciclovir therapy trigger the development of CMV-specific immunity."

Rebecca Kelsey

Original article Reischig, T. et al. Long-term outcomes of pre-emptive valganciclovir compared with valacyclovir prophylaxis for prevention of cytomegalovirus in renal transplantation. J. Am. Soc. Nephrol. doi:10.1681/ ASN.2012010100 